\title{
FACTORES INFLUYENTES EN LA CULMINACIÓN ACADÉMICA DE LA LICENCIATURA EN ADMINISTRACIÓN DE LA UNNE
}

FACTORS THAT INFLUENCE THE ACADEMIC COMPLETION OF THE BACHELORS IN ADMINISTRATION AT UNNE

CARLOS ESTEBAN BONDAR MARÍA GABRIELA LATORRE

MARÍA SILVANA MARTÍNEZ

JORGE GUILLERMO ODRIOZOLA

Facultad de Ciencias Económicas

Universidad Nacional del Nordeste

ARGENTINA 


\title{
RESUMEN
}

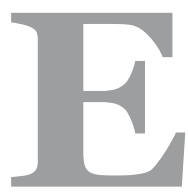

l objeto de estudio analizado en este texto fue el tiempo de permanencia en carácter de estudiantes de los licenciados en administración de la facultad de Ciencias Económicas de la Universidad Nacional del Nordeste, y los motivos que generaron retrasos en su graduación. Como objetivo, se planteó medir el efecto que tuvieron un conjunto de variables explicativas clasificadas en factores demográficos, vocacionales, curriculares y extracurriculares, estas últimas subclasificadas como de índole personal, familiar y laboral, sobre el tiempo de permanencia en la carrera que registraron los graduados encuestados. La investigación actualmente en curso, es de tipo descriptiva, de campo, con obtención de datos primarios a través de encuestas en línea. Se utilizó un cuestionario diseñado con la herramienta Google Drive, y se remitió a los graduados entre los años 2010 y 2013. Las respuestas obtenidas para el presente análisis procedieron de 65 licenciados en administración, sobre una población total de 142. Se observó que factores de naturaleza diversa influyeron sobre el tiempo de permanencia en la carrera. Y una elección tardía de su futura profesión, ya sea porque al ingresar a la facultad el graduado no tenía definida su vocación, o porque la descubrió tras cambiar de carrera, constituyó el principal factor generador de retrasos.

Palabras clave: graduados, profesiones, duración de carrera, vocación.

\begin{abstract}
The object of study analyzed in this work was the time of permanence as students in the case of the graduates in Administration of the Economics Faculty at the National University of the Northeast, and the reasons that led to delays in their academic completion. The main objective was to measure the effects that a set of explanatory variables had. They were classified as: demographic, vocational, curricular, and extracurricular factors, being the latter sub classified as personal, family life, and work types, and considering the length of permanence in the career of the graduates polled. The present research is a descriptive and fieldwork type, with primary data gathered through electronic surveys. It was used an online questionnaire designed with the Google Drive tool and it was sent to the graduates from 2010 to 2013. The answers obtained for the analysis belong to 65 graduates in Administration, out of a total population of 142. It was observed that different factors had an influence on the time of permanence in the career. It was also found out that the main factor that caused delays was a late career choice: either because the graduate had no defined vocation at the time of starting the career, or because he chose it after changing from another career.
\end{abstract}

Keywords: graduates, professions, career duration, vocation. 


\section{INTRODUCCIÓN}

Una de las problemáticas actuales de la educación superior se ubica en torno al periodo de tiempo que deben durar las carreras universitarias, habiendo una tendencia en los países desarrollados, de acortar los planes de estudio a una duración teórica no superior a cuatro (4) años.

Esto puede observarse por ejemplo, en las medidas adoptadas en el Espacio Europeo de Educación Superior (EEES), siendo una de ellas, establecer una estructura de estudios oficiales universitarios de dos ciclos, uno de grado y otro de posgrado, donde el formato de título de grado único, cuenta con una duración de cuatro años.

En Argentina, la mayoría de las carreras universitarias plantean estructuras curriculares de cinco (5) años. Representan un caso particular de análisis las licenciaturas, donde si bien es habitual encontrarse con planes de estudios quinquenales, el trabajo final suele demandar un plazo adicional y no siempre se lo computa en el tiempo total requerido por el estudiante para culminar la carrera.

La Licenciatura en Administración, carrera perteneciente a la Facultad de Ciencias Económicas de la Universidad Nacional del Nordeste (UNNE), plantea una estructura curricular (plan 1998) con una extensión teórica de cinco (5) años. Por lo cual, no resulta ajena a los lineamientos generales de las políticas universitarias establecidas en el país.

Sin embargo, en la práctica, a este periodo debe añadírsele un año más que requieren los estudiantes para completar el trabajo final de graduación, consistente en un proceso de investigación bajo la modalidad de tesina.

En esta misma unidad académica, desde el año 2014, y hasta el 2017 inclusive, viene desarrollándose un proyecto de investigación denominado "Inserción laboral de los contadores públicos, licenciados en administración y en economía de la Universidad Nacional del Nordeste (UNNE)" ${ }^{1}$

Datos emergentes de dicho proyecto demuestran que existen factores de diversa naturaleza que extienden el tiempo de permanencia en la carrera, retrasando la graduación de los estudiantes. Este tiempo no es homogéneo entre los individuos analizados, y además, se evidencia una diferencia entre la duración teórica y la duración media real de la carrera, lo cual ha despertado entre los integrantes del proyecto de investigación el interés en identificar los motivos que las generaron. 


\section{SONDEO PRELIMINAR EN BASES DE DATOS DE LA CARRERA DE ADMINISTRACION}

Si se observa la edad de ingreso, la mayoría de los graduados encuestados ingresó a la carrera en edades muy próximas. La media aritmética es de 19 años; el desvío típico, de 2,9 años; y la edad más frecuente o valor modal, de 18 años.

Pero si se comparan las brechas entre las edades de ingreso a la carrera y de egreso, se detectan variaciones entre los encuestados (figura 1), con un tiempo de permanencia que va desde 5 años y dos meses hasta 18 años, siendo la media aritmética de 9 años y cuatro meses. El índice de retraso es de 1,86.

Figura 1: Edades de ingreso y egreso de los encuestados de la carrera de Licenciatura en Administración de la UNNE, ordenadas por tiempo de permanencia en la Facultad

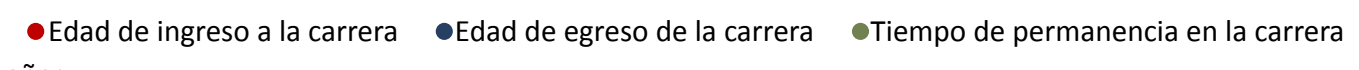
años

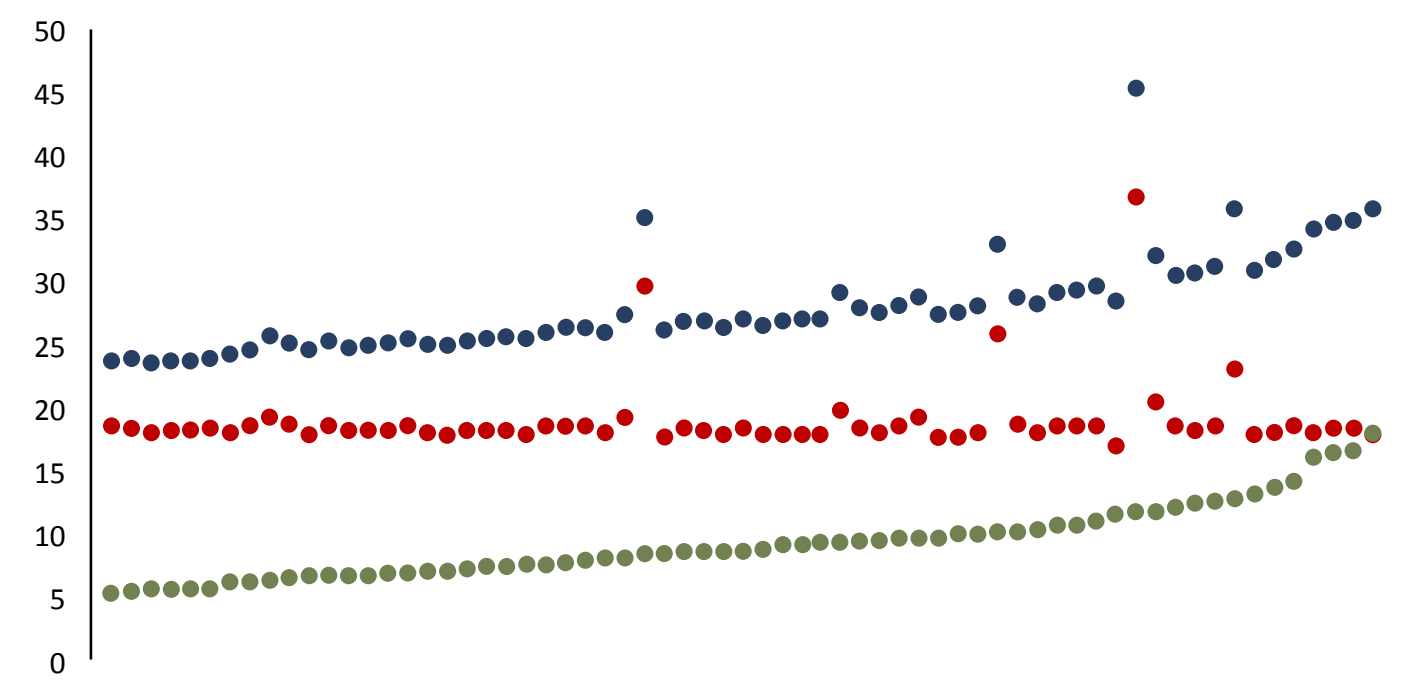

Fuente: elaboración propia

La figura 2 expone en qué proporción se fueron graduando los estudiantes, según la cantidad de años que les llevó el desarrollo de su carrera. 
Figura 2: Tasa de graduación de los encuestados, de acuerdo a los años requeridos para recibirse

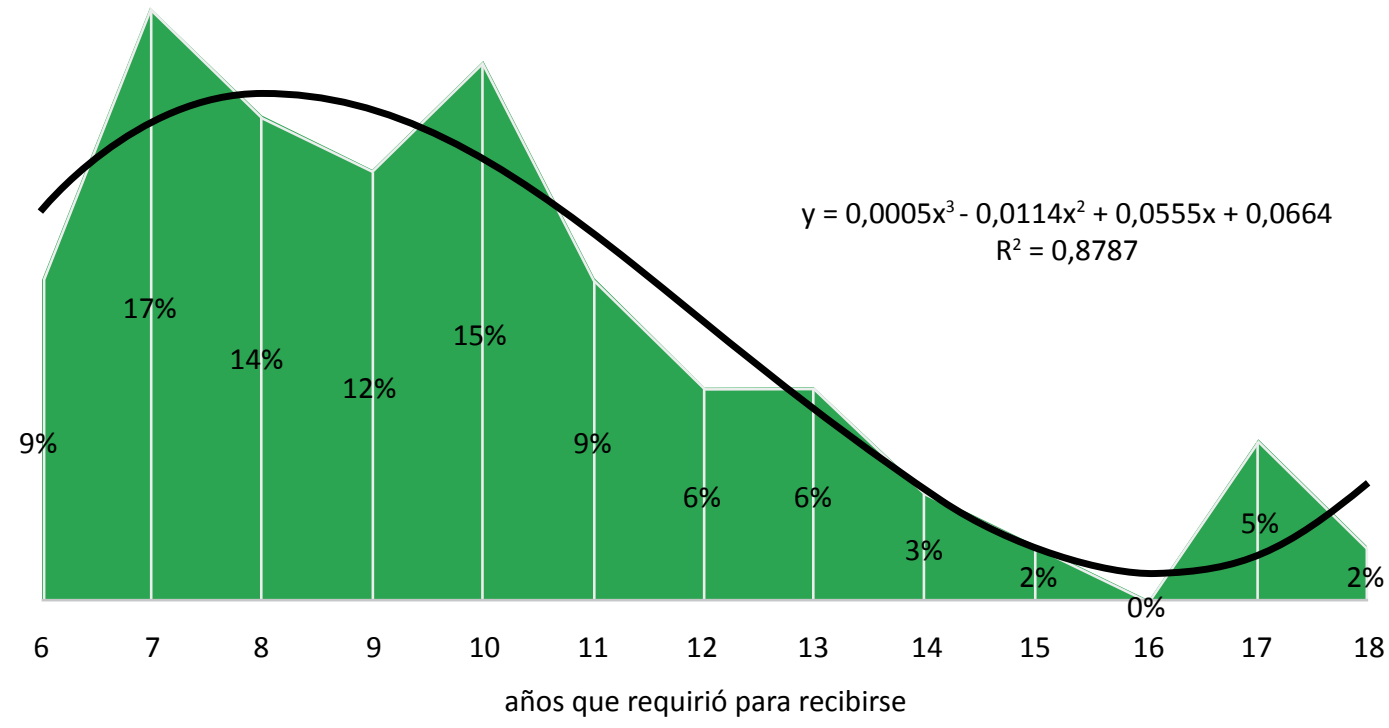

Fuente: elaboración propia

\section{ANTECEDENTES CONSULTADOS}

Vicente Atela (2014), decano de la Facultad de Ciencias Jurídicas y Sociales de la Universidad Nacional de La Plata, expresa que el modelo de estudiante universitario que solamente estudia, es un porcentaje minoritario, y aquel que en los primeros años no trabaja, cuando llega a una instancia avanzada de la carrera comienza a buscar un empleo para poder sostener sus estudios.

De manera particular, la extensión se ve afectada por el proceso de entrada al mercado de trabajo. Ya sea para adquirir experiencias previas, para independizarse de sus padres, por cuestiones económicas o familiares, el $57 \%$ de los licenciados en administración encuestados, accedió un empleo antes de su graduación.

Estas primeras trayectorias laborales tomaron la forma de pasantías, becas, o trabajos en relación de dependencia en puestos de baja jerarquía, para obtener experiencias e ir escalando progresivamente en el competitivo mundo laboral.

Miriam Aparicio (2009), de la Universidad de Cuyo, enfatiza que son las representaciones sociales que los estudiantes tienen sobre el valor de la educación universitaria, un factor preponderante a la hora de culminar sus estudios en el tiempo establecido. Existe una relación indirecta, ya que quienes le dan mayor valoración a la educación superior como canal de progreso, demoran menos en acceder a su titulación universitaria. 
Simultáneamente esta autora agrega que los mercados de trabajo resultan cada vez más conflictivos y que es posible observar una devaluación de las titulaciones o diplomas. Frente a esto, el estudiante que consigue un trabajo que lo deje relativamente satisfecho opta por extender sus estudios y disminuye sus expectativas en relación a obtener un título universitario.

En otro orden Zandomeni, Pacífico, Pagura, Canale, \& Nessier (2011), mencionan que:

\begin{abstract}
$\mathrm{Al}$ indagar sobre las trayectorias académicas, y particularmente las causas que intervinieron en la prolongación de sus estudios, las personas encuestadas manifestaron diferentes motivos entre los que se reconocieron en primer término los laborales, seguido por los motivos asociados a los acontecimientos familiares (matrimonio, hijos, mudanzas, etc.). Por último, las situaciones vinculadas a la carrera como la carga horaria, la extensión e intensidad de los estudios aparecieron como el tercer conjunto de aspectos que operó en el aplazamiento de los estudios (p.27).
\end{abstract}

Resulta importante contextualizar la problemática de carreras con duración media real superior a la duración teórica. En ese sentido, Ríos (2007) reseña para el caso de las licenciaturas en administración dictadas en el país, que en el año 1999 se registró el índice más bajo de retraso en la Universidad San Juan Bosco, equivalente a 1,2; y el más alto en la Universidad de Cuyo con 1,8.

Este índice se obtiene como cociente entre la duración media real y la duración teórica indicada por el plan de estudios de la carrera. Si ambos son iguales, el cociente debe ser igual a 1. Si es mayor, implica demoras entre los estudiantes para graduarse. El cociente 2 indica que el plazo promedio para recibirse duplica al plazo teórico.

\title{
DEFINICIÓN DE FACTORES PROLONGADORES DEL TIEMPO DE PERMANENCIA EN LA CARRERA DE ADMINISTRACIÓN
}

Muchos son los factores que pueden afectar positiva o negativamente al estudiante durante el desarrollo de su carrera, y guardan relación tanto con condiciones extrínsecas a él, como intrínsecas. Entre las extrínsecas se hallan las condiciones económicas, demográficas, sociales y culturales, y entre las intrínsecas, las que se corresponden con el plano psicológico y vocacional del alumno.

En consecuencia, las posibilidades de recibirse varían respecto del plazo establecido como duración teórica de la carrera, referida como cantidad de años requerida de acuerdo a los planes de estudios para graduarse. Usualmente, el estudiante necesita por lo general, un tiempo mayor. 
Dada la diversidad de variables que se consideraron en la encuesta, fue conveniente agrupar los factores en función de su naturaleza. De esta manera se los distinguió entre demográficos, vocacionales, curriculares y extracurriculares. Estos últimos, subclasificados a su vez, en factores condicionantes de índole personal, familiar, o laboral.

En primer lugar, dentro de los factores demográficos, se agruparon las variables: sexo del graduado y orientación hacia las Ciencias Económicas de su título de nivel medio.

En segundo término, como factores vocacionales, se consideraron a las variables: elección de la carrera a temprana edad y cambio de carrera durante la vida universitaria.

Respecto del momento de la elección, se tomó como temprana edad a aquellas respuestas que indicaron durante el cursado del nivel medio o en el momento de su egreso.

En oposición, los encuestados podían indicar que optaron por la carrera una vez incorporados a la vida universitaria. El hecho de un cambio de carrera implica que han comenzado la etapa universitaria cursando otra, en la misma u otra facultad y/o universidades distintas, y en un momento posterior, recién se decidieron por seguir la Licenciatura en Administración.

En tercer lugar, se tomaron como factores curriculares a las variables: realización de la tesina y la aprobación de materias específicas del plan de estudios. Debe entenderse a las mismas como asignaturas con contenidos difíciles de aprobar o con un régimen de correlatividades que afectan el cursado o aprobación de otras de años superiores, retrasando el normal desempeño curricular.

Por último, los factores extracurriculares incluyeron motivos y circunstancias de índole personal, familiar, o laboral, provocando interrupciones o demoras. La interrupción implicó que los alumnos dejaran de cursar por un lapso de tiempo de meses o años y luego retomaran sus estudios.

Por otra parte, se consideró retraso o demora, cuando el ritmo normal de estudio se desaceleró, verificable a través de la disminución en la cantidad de materias aprobadas por año, según la propuesta del plan de estudios.

\section{ASPECTOS METODOLÓGICOS}

Los resultados expuestos surgen del proyecto de investigación descripto al inicio, y actualmente en desarrollo. Se trata de un estudio de diseño descriptivo, de campo, donde los datos primarios se obtuvieron mediante la aplicación de un instrumento estructurado, bajo la modalidad de encuesta en línea.

Se utilizó un cuestionario diseñado en Google Drive, que recabó datos de los graduados entre los años 2010 y 2013 de las carreras Contador Público, Licenciatura en Administración y 
Licenciatura en Economía, pertenecientes a la Facultad de Ciencias Económicas de la UNNE, referidos a su inserción y trayectoria laboral, su formación de grado y de posgrado, y su relación con los Consejos Profesionales de Ciencias Económicas.

Para esta investigación, se definió como población de estudio al conjunto de licenciados en administración, egresados entre los años indicados.

El tamaño de la población fue de 142 profesionales, y la muestra no probabilística por conveniencia en función de las primeras respuestas obtenidas, ascendió a 65, lo que representa un $46 \%$ del total de la población. Entre los encuestados, el 37\% registró ser de sexo masculino.

\section{ANÁLISIS DE DATOS}

Para identificar los factores que provocaron retrasos en la graduación de los licenciados en administración, se aplicó la técnica estadística de dependencia, denominada Análisis de Clasificación Múltiple (ACM) utilizando el software Dyane versión 4 del catedrático español Santesmases Mestre (2009).

Según Malhotra (2008), las técnicas de dependencia permiten medir el efecto de un conjunto de variables clasificadas como explicativas o independientes, sobre otras consideradas dependientes.

En particular, el ACM se caracteriza por permitir el uso de variables explicativas de naturaleza no métrica, es decir aquellas que se miden en escala nominal u ordinal. Por ejemplo, una pregunta utilizada fue: ¿Trabajó mientras estudiaba en la Facultad? Y sus posibles respuestas: sí y no.

Para este análisis, se definió como variable dependiente, al tiempo de permanencia de los graduados en la carrera. La unidad de medida utilizada para esta variable fue la cantidad de años que requirieron para recibirse desde su ingreso a la Facultad de Ciencias Económicas, hasta defender la tesina.

Tabla 1: estadísticos descriptivos de la muestra de graduados encuestados

\begin{tabular}{cccc}
\hline Variable criterio: & \multicolumn{3}{c}{ Tiempo de permanencia de los graduados en la carrera } \\
\hline Media (gran media) & 9,3 (años) & Suma de cuadrados total & 552,46 \\
Desviación estándar & 2,9 (años) & Suma de cuadrados explicada & 391,01 \\
Número de casos & 65 & Proporción de varianza explicada & 0,71 \\
\hline
\end{tabular}

Fuente: elaboración propia

Los factores analizados explican el 71\% de la varianza de la variable dependiente (tabla 1).

En la tabla 2, se expone el conjunto de variables consideradas inicialmente explicativas, junto con los coeficientes estadísticos eta cuadrado, o como define Requena Santos (2000), proporción de la varianza explicada por el predictor sobre la variable dependiente, y beta cuadrado, o fuerza de cada relación manteniendo constantes los efectos de las restantes variables. 
Asimismo, se deduce que las interrupciones por motivos laborales, familiares, y personales; el cambio de carrera; el desarrollo de una actividad laboral durante el cursado; la realización de la tesina; las dificultades con las correlatividades o con la aprobación de algunas materias del plan de estudio; y asuntos familiares que demandaron tiempo durante el cursado, constituyeron los factores más relevantes que influyeron provocando demoras en los graduados para recibirse y no culminar la carrera en los tiempos teóricos establecidos según el plan de estudio.

Tabla 2: coeficientes eta cuadrado y beta cuadrado de las variables explicativas

\begin{tabular}{lccc}
\hline \multicolumn{1}{c}{ Variables explicativas } & Clasificación & Eta cuadrado & Beta cuadrado \\
\hline Sexo & Demográfico & 0,0002 & 0,0006 \\
Título de nivel medio orientado & Demográfico & 0,0060 & 0,0114 \\
Elección de la carrera en el nivel medio & Vocacional & 0,1687 & 0,0067 \\
Interrupción por motivos personales* & Extracurricular & 0,2347 & 0,0226 \\
Interrupción por motivos familiares* & Extracurricular & 0,1792 & 0,0394 \\
Interrupción por motivos laborales* & Extracurricular & 0,0570 & 0,0455 \\
Cambio de carrera* & Vocacional & 0,2834 & 0,1762 \\
Actividad laboral durante la carrera* & Extracurricular & 0,1180 & 0,0398 \\
Vinculación entre trabajo y estudio & Extracurricular & 0,1126 & 0,0031 \\
Retraso en la carrera por la tesina* & Curricular & 0,0034 & 0,0359 \\
Retraso... por dificultades con las materias* & Curricular & 0,0119 & 0,0256 \\
Retraso... por viajes & Extracurricular & 0,0716 & 0,0057 \\
Retraso... por asuntos familiares* & Extracurricular & 0,1179 & 0,0679 \\
Retraso... por motivo laboral & Extracurricular & 0,0066 & 0,0115 \\
\hline
\end{tabular}

Fuente: elaboración propia

A efectos de apreciar gráficamente estas relaciones mediante tabulaciones cruzadas, se convirtió la variable métrica "tiempo de permanencia de los graduados en la carrera" en no métrica, utilizando una escala interválica de cinco descriptores o categorías.

Las categorías así obtenidas fueron 6,5 años o menos; 6,6 a 8,1 años; 8,2 a 10,7 años; 10,8 a 14,2 y más de 14,2 años de permanencia en la carrera.

Tabla 3: coeficientes chi cuadrado y $p$ de las variables explicativas, obtenidos a través del cruzamiento con la variable criterio, convertida a no métrica

\begin{tabular}{lcc}
\hline Variables explicativas (nominales con respuestas sí y no) & Chi cuadrado (GL 4) & P \\
\hline Sexo masculino & 4,8502 & 0,3030 \\
Título de nivel medio orientado & 8,6338 & 0,0709 \\
Elección de la carrera en el nivel medio & 15,5821 & 0,0036 \\
Interrupción por motivos personales & 18,0570 & 0,0012 \\
Interrupción por motivos familiares & 14,2339 & 0,0066 \\
Interrupción por motivos laborales & 8,5290 & 0,0740 \\
Cambio de carrera & 24,5113 & 0,0001 \\
Actividad laboral durante la carrera & 15,8227 & 0,0033 \\
Vinculación entre trabajo y estudio & 9,8712 & 0,0427 \\
\hline
\end{tabular}


Tabla 3: coeficientes chi cuadrado y p de las variables explicativas, obtenidos a través del cruzamiento con la variable criterio, convertida a no métrica

\begin{tabular}{lcc}
\hline Variables explicativas (nominales con respuestas sí y no) & Chi cuadrado (GL 4) & P \\
\hline Retraso en la carrera por la tesina & 3,4289 & 0,4888 \\
Retraso... por dificultades con las materias & 7,8557 & 0,0970 \\
Retraso... por viajes & 9,3968 & 0,0519 \\
Retraso... por asuntos familiares & 8,1500 & 0,0862 \\
Retraso... por motivo laboral & 5,5554 & 0,2349 \\
\hline
\end{tabular}

Fuente: elaboración propia

En cada tabulación cruzada se realizó el análisis chi cuadrado para determinar si las diferencias observadas resultan estadísticamente significativas (p igual o inferior a 0,05).

\section{INFLUENCIA DE FACTORES DEMOGRÁFICOS Y VOCACIONALES}

Las variables sexo del graduado y titulación de nivel medio orientado a las Ciencias Económicas no produjeron efectos significativos. De esta manera, se descartan los factores demográficos entre los considerados como explicativos del retraso en la graduación de los encuestados.

Por otra parte, en la Facultad de Ciencias Económicas de la UNNE, el ingresante cursa durante el primer año un ciclo básico común para las carreras de Contador Público, Licenciatura en Administración y Licenciatura en Economía; y a partir del segundo año, opta por una de esas carreras para graduarse.

La decisión de seguir la Licenciatura en Administración a temprana edad, mientras se encontraba en el nivel educativo medio o al momento de su finalización influenció favorablemente, ya que en este grupo la mayoría se recibió a término, comparando con aquellos graduados que eligieron la carrera tras ingresar a la universidad.

Siguiendo este análisis, aquellos egresados que realizaron un cambio de carrera, es decir que abandonaron otra para optar por la Licenciatura en Administración, tardaron más tiempo en finalizar sus estudios.

Así, un 70\% de los que se decidieron cuando cursaban el nivel medio (escuela secundaria), terminó la Licenciatura en un plazo no mayor a 6 años y medio. Por el contrario, aquellos que lo hicieron en la etapa universitaria (habiendo iniciado el Ciclo Básico Común en Ciencias Económicas sin tener una inclinación específica a la carrera, o realizaron otras carreras con anterioridad), demoraron más en recibirse. 
Figura 3: proporción de graduados que eligió seguir la carrera durante el cursado del nivel medio y de graduados que cambiaron de carrera en la etapa universitaria, según la cantidad de años que requirieron para recibirse

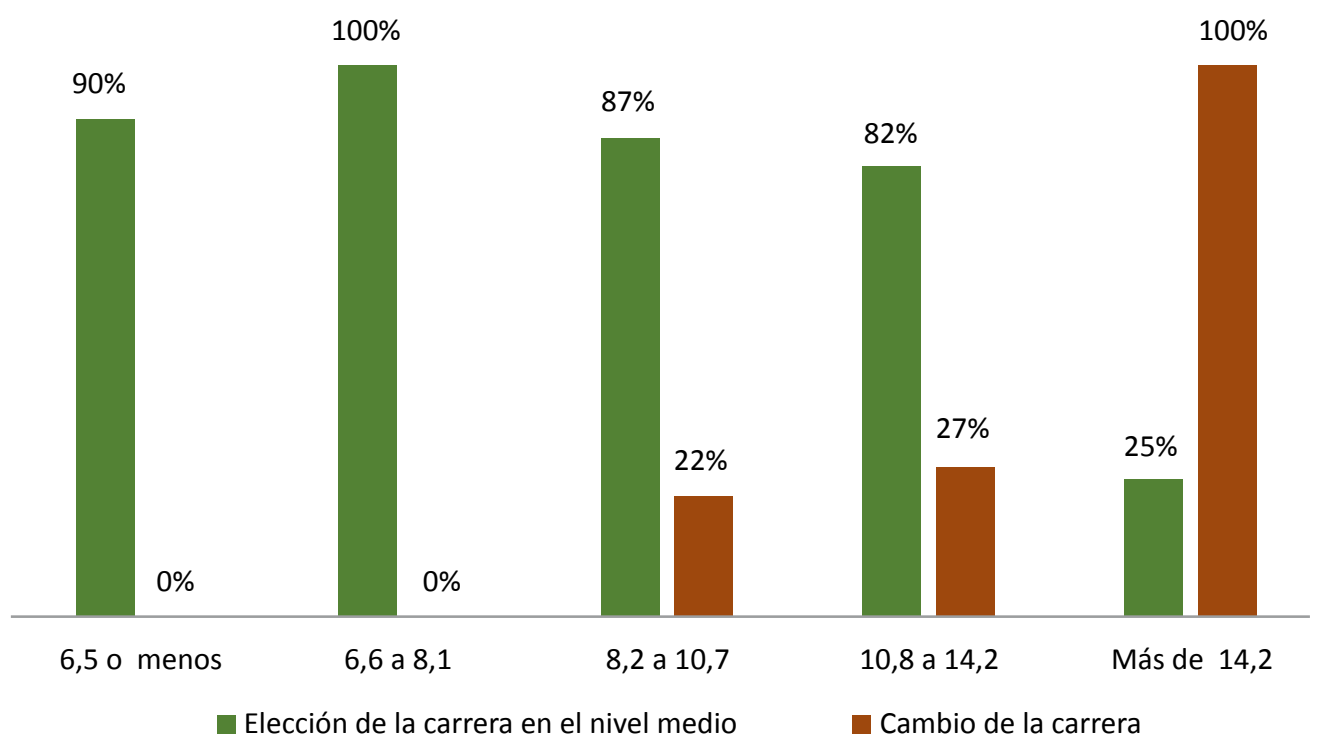

Fuente: elaboración propia

Sin embargo, si se analiza la orientación de los títulos de nivel secundario, no resulta posible establecer una relación entre este factor y una extensión en el tiempo de permanencia en la carrera.

El 65\% de los graduados encuestados que indicaron poseer título secundario orientado a las Ciencias Económicas terminaron la carrera en el plazo que va desde más de 8,1 a 10,7 años.

En este sentido, disponer de un título orientado no facilitó al alumno el desarrollo de la carrera a término.

\section{INFLUENCIA DE FACTORES CURRICULARES}

Puede observarse que la realización de la tesina demanda un tiempo adicional no contemplado en la duración teórica de cinco (5) años establecida por el plan de estudios vigente (plan 1998). 
Figura 4: proporción de graduados que tuvo retrasos originados en la realización de la tesina o en dificultades para aprobar materias específicas del plan de estudios, según la cantidad de años que requirieron para recibirse

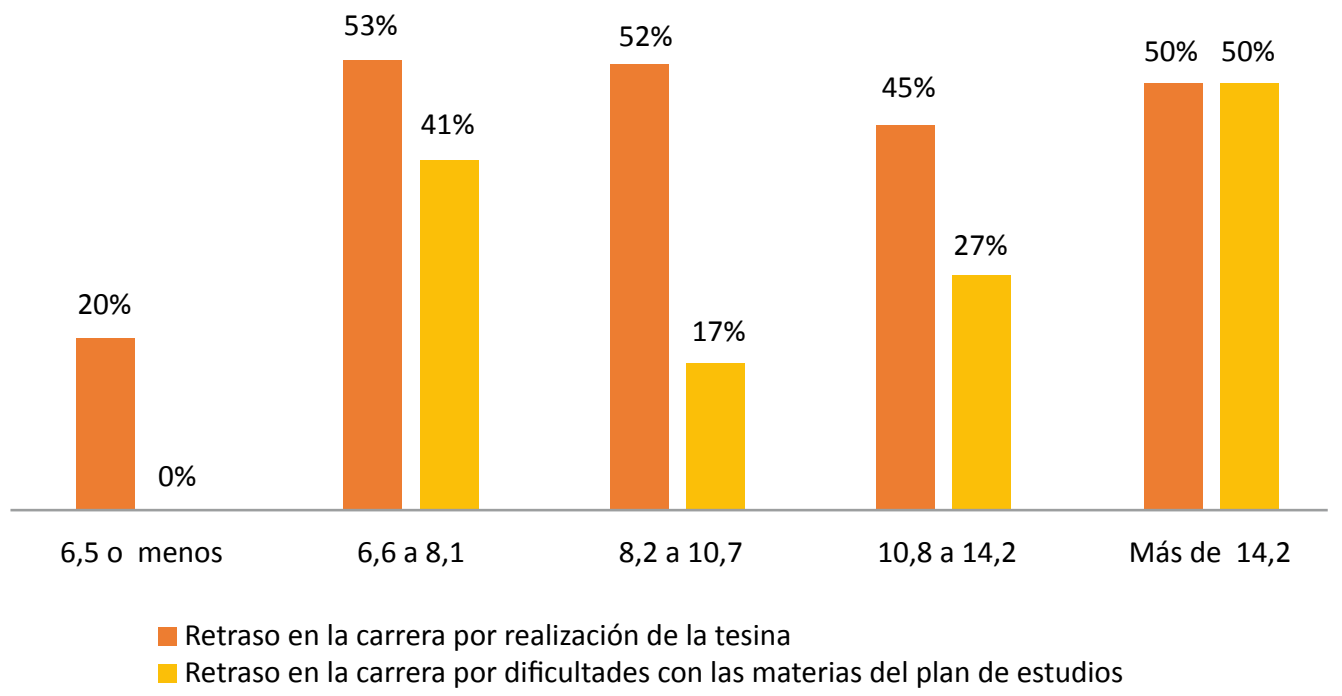

Fuente: elaboración propia

Aproximadamente, un año y medio $(1,5)$, es el tiempo promedio que normalmente demanda la conclusión de esta modalidad de trabajo final en la carrera, de acuerdo a registros de la asignatura a cargo del proceso.

De acuerdo a opiniones de los encuestados, las demoras en una parte de ellos, se debieron tanto a la tesina como a las materias que requerían contar con las correlatividades al día, o que resultaban de contenido de difícil.

Sin embargo, no es posible establecer una relación directa entre estas variables y el tiempo de permanencia en la carrera.

\section{INFLUENCIA DE FACTORES EXTRACURRICULARES QUE GENERARON DEMORAS}

Los asuntos familiares, y en menor medida los motivos laborales, provocaron demoras para recibirse entre los graduados encuestados. 
Figura 5: proporción de graduados que tuvo retrasos originados en viajes, asuntos familiares, o por motivos laborales, según la cantidad de años que requirieron para recibirse

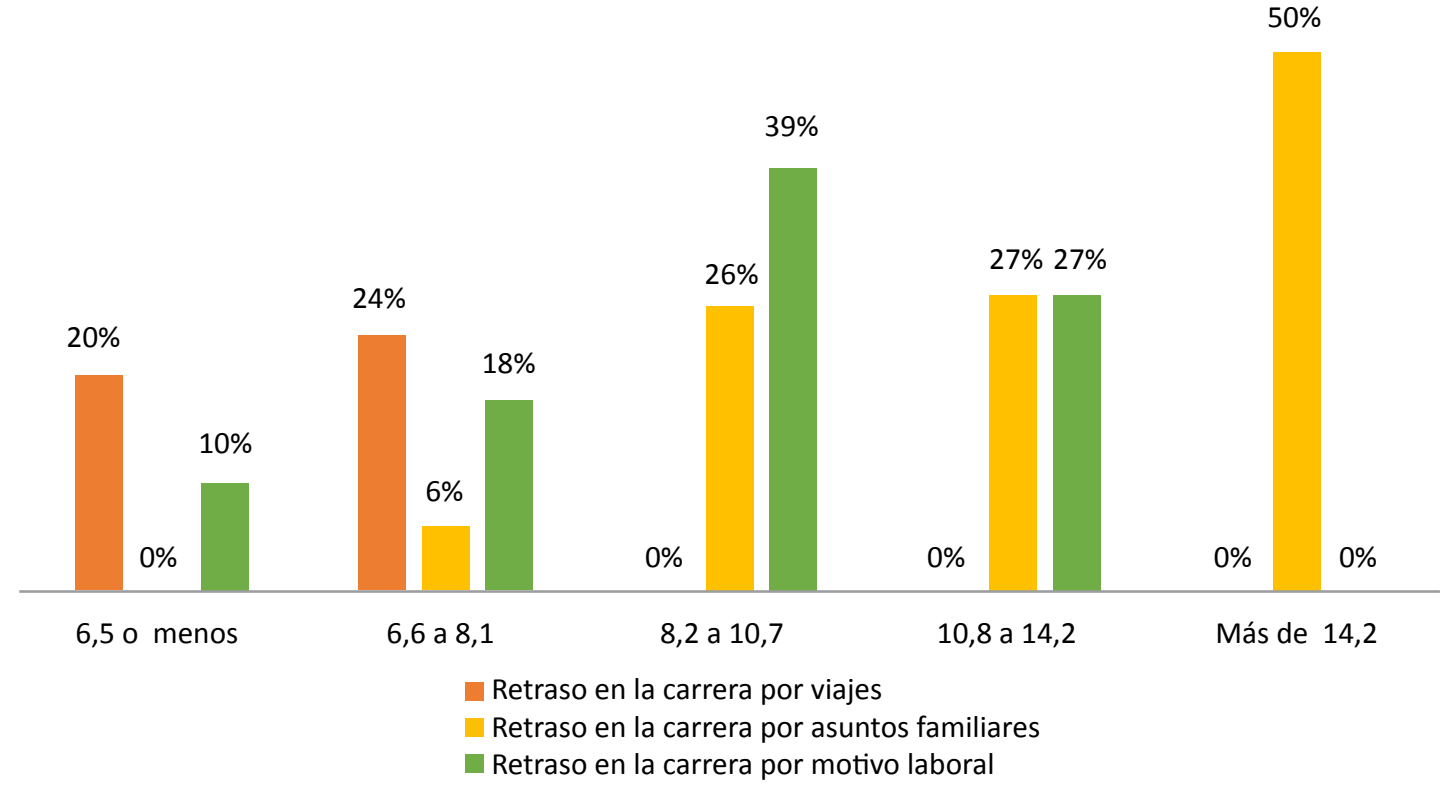

Fuente: elaboración propia

Los viajes sólo afectaron a una parte reducida de la muestra, lo cual se debió a pasantías de trabajo en el extranjero o becas internacionales, en la mayoría de los casos.

Los asuntos familiares (los que incluyen razones de salud propia o de algún familiar, problemas económicos y la formación de su propio núcleo familiar) son los que influyeron en mayor medida, su relevancia se acrecienta en relación directa con la cantidad de años de retraso para culminar los estudios, siendo el único factor determinante en los que tardaron más de catorce (14) años para su graduación.

Por último, se puede observar que los retrasos por motivos laborales se identifican en la mayoría de las categorías propuestas, demostrando que es alto el porcentaje de graduados que obtienen su empleo antes de su graduación, identificando a este factor como un limitante a la hora de graduarse en los tiempos definidos por los planes de estudios.

\section{INFLUENCIA DE LA ACTIVIDAD LABORAL}

El desarrollo de la actividad laboral durante la época de estudio debe tomarse también como un factor extracurricular. 
Figura 6: proporción de graduados en actividad laboral y mención de vinculación entre su trabajo y la carrera estudiada, según la cantidad de años que requirieron para recibirse

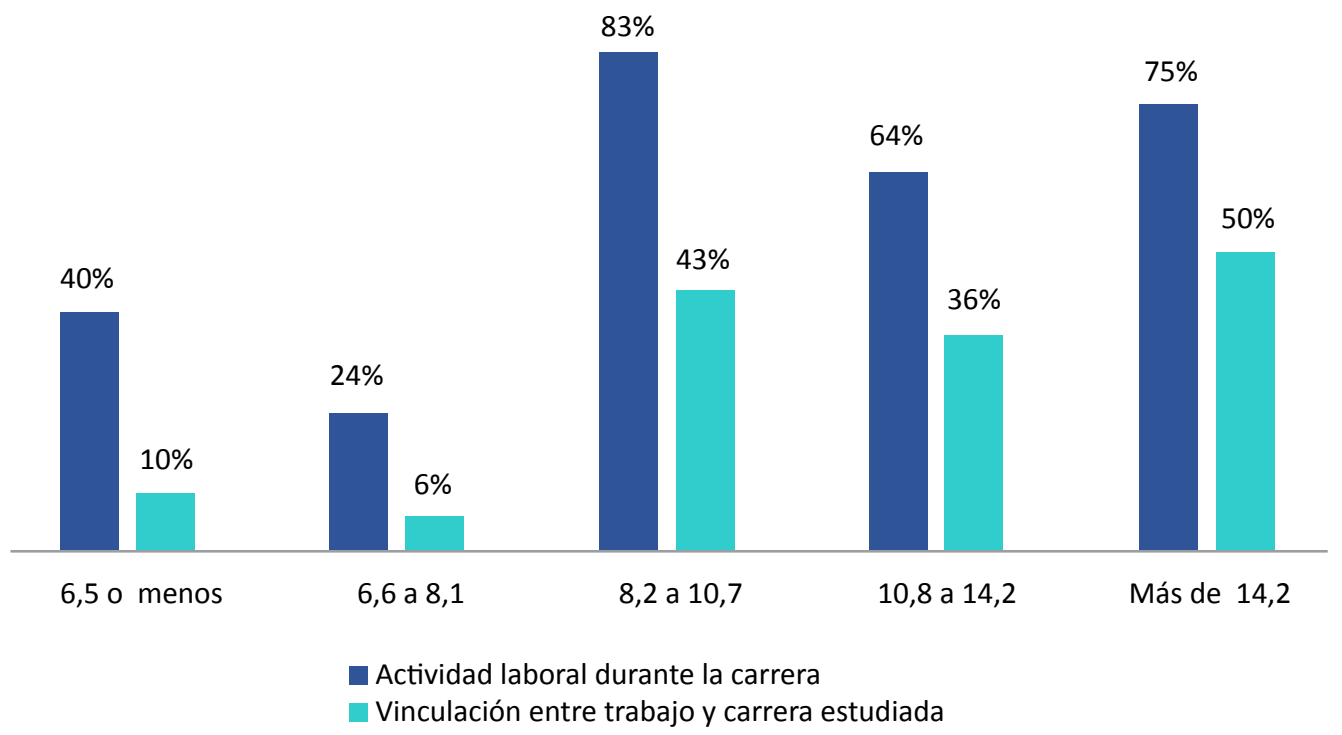

Fuente: elaboración propia

Si bien se observa un efecto de prolongación sobre los años que requirió el encuestado para recibirse, lo llamativo es que a mayor vinculación entre el puesto de trabajo y la formación que brinda la carrera cursada, mayor fue la demora experimentada. Esto puede vincularse a lo expuesto en uno de los antecedentes consultados, donde la satisfacción con el trabajo "relaja” al estudiante e impide que avance para alcanzar la titulación.

\section{INFLUENCIA DE FACTORES EXTRACURRICULARES QUE GENERARON INTERRUPCIONES}

Cabe recordar que las interrupciones implicaron el abandono transitorio del cursado de la carrera, por lo cual se asume que el impacto habrá sido más fuerte en el graduado desde lo actitudinal. Significa que el retomar los estudios luego de un tiempo, habrá requerido en la mayoría de los casos, un esfuerzo sicológico o incentivo mayor. 
Figura 7: proporción de graduados que interrumpió el cursado de la carrera por motivos personales, familiares, o laborales, según la cantidad de años que requirieron para recibirse

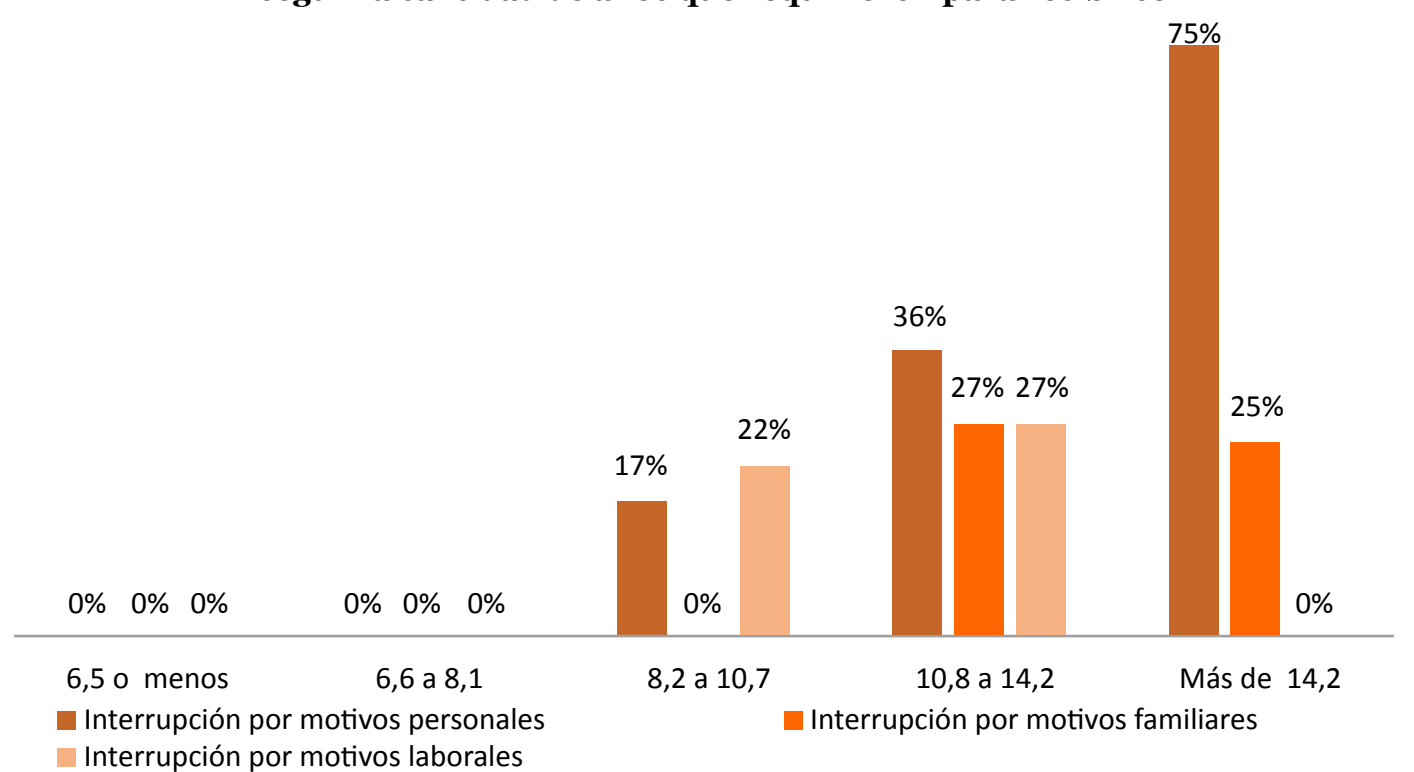

Fuente: elaboración propia

A mayor tiempo de permanencia en la carrera, se registraron más respuestas de interrupciones durante el cursado. Los encuestados que expresaron haber tenido interrupciones alcanzaron el $28 \%$ de la muestra, perteneciendo a los grupos que tuvieron una permanencia en la carrera superior a los 8,1 años.

En función del número de respuestas, los motivos pueden ordenarse de menor a mayor en familiares, laborales y personales. Entre las causales bajo análisis, las de índole personal afectaron en mayor medida, a aquellos graduados que más tardaron en recibirse.

Los motivos familiares (22\%), se observaron entre los graduados con permanencia en la carrera superior a los 10,7 años. Los motivos laborales (44\%), se concentraron entre los grupos con permanencia superior a 8,1 e inferior a 14,2 años. Y los motivos personales (61\%), se registraron en las categorías de permanencia mayores a los 8,1 años, relacionándose en forma directa con la extensión de la misma.

\section{CONCLUSIONES}

De acuerdo con el plan vigente, se espera que un alumno de la Licenciatura en Administración de la UNNE se reciba en el término de cinco (5) años. No obstante, las mayores tasas 
de graduación entre los encuestados, se registraron desde los siete (7) hasta los diez (10) años de permanencia en la carrera.

Interrupciones motivadas en cuestiones laborales, familiares, o personales; el desarrollo de una actividad laboral durante el cursado de la carrera; la realización de la tesina; las dificultades con las correlatividades o con la aprobación de algunas materias del plan de estudios; y demoras por asuntos personales y familiares, fueron los factores más relevantes que demandaron, en estos egresados, más tiempo para finalizar sus estudios que el plazo establecido en la estructura curricular.

En base a los datos analizados, puede aseverarse que la elección tardía de la carrera, ya sea porque al ingresar a la facultad los graduados no tenían definida su vocación, o porque la descubrieron tras cambiar de carrera, constituyó el factor principal generador de estos retrasos. Asimismo, la temprana inserción al mercado de trabajo actuó como segundo factor en orden de importancia. En tercer lugar, la atención de asuntos personales o familiares.

De esta manera, se observa que el factor vocacional actúa como impulsor en el estudiante, motivándolo para que avance en la carrera y facilitándole las posibilidades para obtener una graduación a término.

Es común denominador de las carreras que se dictan en Argentina, que los tiempos promedios de graduación sean mayores a los establecidos, provocando un desgaste en el estudiante. $\mathrm{Y}$ en ocasiones, hasta opta por abandonar su carrera a fin de desarrollarse laboralmente.

Por lo tanto, a nivel de políticas universitarias, es necesario repensar si los planes de estudios se ajustan a las posibilidades reales que tienen los estudiantes de recibirse en la cantidad de años establecida como duración teórica.

\section{REFERENCIAS BIBLIOGRÁFICAS}

Aparicio, M. (2009) La demora en los estudios universitarios. Tomo I: Causas de una perspectiva cuantitativa. Mendoza, Argentina: EDIUNC.

Atela, V. (2014, 24 de agosto) En la Universidad platense, los alumnos tardan más de ocho años en recibirse. El Día. Recuperado de http://pasado.eldia.com/edis/20140824/En-Universidadplatense-alumnos-tardan-mas-ocho-anos-recibirse-laciudado.htm

Malhotra, N. (2008) Investigación de mercados. México: PEARSON EDUCATION.

Oficina de Información al Alumnado de la Universidad de Alicante (s.f.) Espacio Europeo de Educación Superior. Recuperado de https://web.ua.es/es/oia/preguntas/espacio-europeode-educacion-superior.html 
Requena Santos, F. (2000). Sociedad, Cultura y Desarrollo: apuntes para un análisis comparado entre España y los Estados Unidos. Almería, España: Universidad Almería.

Ríos, G. (octubre y noviembre, 2007). Duración real de los estudios universitarios: desgranamiento y egreso. En sd., IX Jornadas Argentinas de Estudios de Población. Jornadas llevadas a cabo por la Asociación de Estudios de Población de la Argentina, Huerta Grande, Córdoba. Recuperado de http://www.academica.org/ooo-028/40.pdf

Santesmases Mestre, M. (2009). DYANE Versión 4. Diseño y análisis de encuestas en investigación social y de mercados. Madrid, España: Pirámide.

Zandomeni, N., Pacífico, A., Pagura, F., Canale, S., \& Nessier, A. (2011). Estudiantes universitarios avanzados que interrumpieron su carrera: trazos de sus trayectorias académicas y laborales. Ciencias Económicas, 2 (17), 11-30. Recuperado de https://bibliotecavirtual.unl.edu.ar/ ojs/index.php/CE/article/viewFile/4289/6508

\section{CURRICULUM VITAE}

\section{Carlos Esteban Bondar}

Especialista en Gerencia y Vinculación Tecnológica, docente investigador con dedicación exclusiva de la Facultad de Ciencias Económicas de la UNNE.

cebondar@eco.unne.edu.ar

\section{María Gabriela Latorre}

Licenciada en Administración, docente investigadora con dedicación exclusiva de la Facultad de Ciencias Económicas de la UNNE.

mgabrielalatorre@gmail.com

\section{María Silvana Martínez}

Especialista en Gestión Pública Provincial y Municipal, joven investigadora del CECyT (FACP$\mathrm{CE}$ ), personal de apoyo en investigación de la Facultad de Ciencias Económicas de la UNNE. capelucier@hotmail.com

\section{Jorge Guillermo Odriozola}

Magister en Marketing, docente investigador y vicedecano de la Facultad de Ciencias Económicas de la UNNE.

godriozola@eco.unne.edu.ar 